\title{
Interpretation und Weltbezug
}

\author{
Von EMIL ANGEHRN (Basel)
}

HANS KRÄMER: KRITIK DER HERMENEUTIK. Interpretationsphilosophie und Realismus. C. H. Beck Verlag, München 2007, 253 S.

Die Hermeneutik und ihr Anderes: Mehrfach ist in den vergangenen Jahrzehnten Hermeneutik im Verhältnis zu dem, was als ihr Anderes gilt, diskutiert worden - im Verhältnis zur Szientistik, zur Semiotik, zur analytischen Sprachphilosophie, zur Dekonstruktion. Zumeist ging es dabei um ein Anderes, von dem sich entweder die Hermeneutik konzeptuell absetzte oder das sich seinerseits in Opposition zur Hermeneutik definierte. Nicht selten stand indes auch zur Diskussion, wieweit es sich bei den Opposita nicht vielmehr um kompatible, sich ergänzende oder konvergierende Konzepte handelt, wieweit etwa die Dekonstruktion als ein bestimmter Typus hermeneutischer Arbeit, die analytische und die hermeneutische Philosophie als ,komplementäre Spielarten der linguistischen Wende‘ (Habermas) zu betrachten

11 M. Dummett, Thought and Reality, Oxford 2006, VII f. Zur Erläuterung des sozialen und modalen Elements der Rechtfertigung in dieser Bestimmung Dummetts vgl. S. Tolksdorf, Semantik der Vergangenheit, in: Deutsche Zeitschrift für Philosophie, 56 (2008) 1, 35-51.

12 Vgl. L. Moretti, Dummett and the problem of the vanishing past, in: Linguistic and Philosophical Investigations, 2008. 
seien. Solchen Annäherungen lag meist eine affirmativere Sicht der Hermeneutik zu Grunde als den vielfältigen Kontrastierungen.

Die besondere Stoßrichtung der von Hans Krämer vorgelegten Kritik der Hermeneutik in dieser Problemkonstellation ist durch die Verbindung von Titel und Untertitel seiner Abhandlung angezeigt. Diese nähert die Hermeneutik und die ,Interpretationsphilosophie' einander an, indem sie beide einer gemeinsamen Kritik unterwirft. ,Hermeneutik' steht dabei für die moderne Philosophische Hermeneutik, im Besonderen die Theorie Hans-Georg Gadamers; ,Interpretationsphilosophie‘ meint die namentlich durch Hans Lenk und Günter Abel (daneben J. Simon, R. Rorty) vertretene Theorie der Interpretation. ,Kritik' ist in kantischem Sinne als Überprüfung und Einschränkung der Geltungsansprüche einer Wissensform gemeint, wobei die Überprüfung im Ganzen eine unverkennbar ,kritisierende“ Dominante gewinnt: Weniger als um den Aufweis der wissenskonstitutiven Funktion des Verstehens und Interpretierens geht es um die Zurückweisung illegitimer Ansprüche, ja, die basale Inkonsistenz und Falschheit einer bestimmten Theorierichtung. Die Hauptlinie der Kritik gilt der antirealistischen Tendenz, welche Hermeneutik und Interpretationsphilosophie nach Einschätzung des Autors teilen. Im Lichte der Kritik rücken diese Theorien in eine Nähe, die dem Selbstverständnis zumal der Interpretationsphilosophie zuwiderläuft, die sich weithin gegen die Hermeneutik definiert; zum demonstrandum des Buchs gehört damit nicht nur die Triftigkeit der Kritik, sondern auch die Tragfähigkeit dieser zusammenschließenden Diagnose.

$\mathrm{Zu}$ dieser Konvergenz kommt der Verfasser teils durch eine spezifische Akzentuierung der Hermeneutik Gadamers, teils in deren Abhebung von einem umfassenderen, älteren Hermeneutik-Verständnis (das er in neueren Ansätzen bei E. Betti und P. Ricœur aufgehoben sieht); gegen die „szientifische“ Hermeneutik, die „am Realismus des geisteswissenschaftlichen Erkennens auch in der Metatheorie festhält“, schlägt die ,phänomenologische Wendung der Hermeneutik“ in der Nachfolge Heideggers eine ,antirealistische Richtung“ ein (12 f.), die an Verschiedenem festzumachen ist.

Ein auffallendes Merkmal ist die bevorzugte Ausrichtung an der Sprache: Gegenstand der Interpretation sind Zeichen und Texte, nicht die „Auslegung der Sachen selbst“ (14). Die Rede von ,Zeichen“ gewinnt im Interpretationismus eine „erweiterte, ja uneigentliche Bedeutung“, sofern nicht mehr die Verweisung auf die Sache, sondern am Ende „die Beziehung von Zeichen untereinander" zur Diskussion steht (211). Das traditionelle Verständnis der Interpretation - analog dem semiotischen Dreieck von Zeichen, Vorstellung/Bedeutung und (realem) Objekt - wird auf das duale Verhältnis von Zeichen und Bedeutung oder gar den Binnenprozess der Zeichen reduziert. Die Totalisierung der Interpretation geht einher mit der Geschlossenheit eines Verstehens, das nicht mehr durch die Deutung hindurch auf ein Ansichseiendes ausgreift. Diese Immanenz kommt nach Krämer retrospektiv wie prospektiv zum Tragen. Jede Interpretation schließt an vorausgehende Interpretationen an, ohne auf ein „ursprünglich Uninterpretiertes“ (78) zu referieren; nach der Gegenseite verbleibt sie im Binnenraum der Deutens, vollzieht sie Gadamer zufolge ein Andersinterpretieren, ohne sich an einem Kriterium von Objektivität oder Wahrheit auszurichten. Gegen die tendenzielle Gleichwertigkeit aller Lesarten will Krämer die Approximativität als Richtschnur rehabilitieren; in Wahrheit, so seine These, unterscheiden sich Interpretationen nach dem Mehr oder Weniger der Annäherung, des Fortschritts oder Rückschritts in der Explikation der Sache (87, 78). In diesem Punkt steht Derrida näher bei der normalen Intuition des Erkennens als Gadamer, sofern die différance das Moment der Andersheit mit demjenigen des Aufschubs, damit auch einer teleologischen Gerichtetheit verknüpft (69 f.). Umgekehrt sieht Krämer in Gadamers Aufwertung der Anwendung als Kern des Hermeneutischen ein Indiz der antirealistischen Selbstbezüglichkeit eines Verstehens, dem sowohl die Explikation wie die Anwendung zuge- 
schlagen werden, während die traditionellen Momente der Applikation, die den Bezug zur Wirklichkeit wie zur Praxis herstellen, verloren gehen (28).

Auch andere Brennpunkte und Leitbegriffe der Gadamerschen Hermeneutik - Kunst als Paradigma, Interpretation als Übersetzung, hermeneutische Erfahrung, Horizontverschmelzung, Wirkungsgeschichte und anderes - stehen für die subjektivistisch-antirealistische Tendenz, gegen die sich Krämers Kritik richtet. Ein besonderes Gewicht kommt dem Primat der historischen Orientierung zu, wobei nicht der von anderen Autoren monierte Traditionalismus den primären Stein des Anstoßes bildet. Im Zentrum stehen einerseits die einseitige Auffassung des Historischen, anderseits das generelle Überwiegen der historischen gegenüber der systematischen Betrachtung. Gegen die Festlegung der Wirkungsgeschichte auf Linearität betont Krämer andere Prozessformen des Historischen (Emergenz, Spirale, kontingente Verläufe). Vor allem aber beharrt er, gegen die „Verabsolutierung der Geschichte“, darauf, dass Geschichte „nur eine Ordnungsform neben anderen ist“ (34) und dass auch innerhalb hermeneutischer und kulturwissenschaftlicher Betrachtungen das Eigengewicht der systematischen und synchronen Optik gegen die diachrone Orientierung zur Geltung zu bringen ist. Interpretation und Verstehen finden nicht nur im Verhältnis zu vergangenen Dokumenten und Ereignissen, sondern innerhalb des Gegenwartsdenkens und im Untersuchungsfeld systematischer Wissenschaften statt. Insofern ist auch eine Revision der Gewichtung zwischen Natur-, Geistes- und Kulturwissenschaften gegenüber der weithin unterstellten Allianz zwischen Verstehen und Kulturwissenschaft verlangt. Die von Krämer postulierte Gegenlektüre der Hermeneutik ist nicht in das Schema einer Zweiteilung der Kulturen einzuschreiben. Die Lehre vom Verstehen und Auslegen interessiert im Rahmen einer allgemeinen epistemologischen Problemstellung, nicht als Sondermethode bestimmter Disziplinen.

Konkret verbindet sich damit eine Depotenzierung des hermeneutischen Zirkels wie der interpretationistisch-konstruktivistischen Lesart des Wissens. Das Anschließen und Weiterführen in Kultur und Wissenschaft folgt zunächst einer Logik des „Mehrwissens“, in welchem Krämer den ,am besten beglaubigten Sachverhalt des interepochalen und -kulturellen Verhältnisses“ sieht, auf dessen Basis ,,andere Eigenschaften und Folgerungen - etwa Zirkelverhältnisse“ zur Diskussion stehen (142). Die Weiterentwicklung geschieht nicht primär im Modus des Uminterpretierens, sondern des Erwerbs von neuen Kenntnissen und Sichtweisen, die im „quasi-subtraktiven Vergleich“ bei gleichzeitiger „Kontinuität der Bezugnahme“ den konkreten Wissensfortgang anzeigen (143). Die objektive Referenz, welche nicht erst im Medium der Interpretation zu Stande kommt, sondern dieser voraus- und zu Grunde liegt, ist jedoch nicht nur im historischen Rückgriff, sondern ebenso im aktuellen Auslegen von Belang. Nach Krämer widerspricht ein radikaler Interpretationismus, der den Gegenstand wie das Kategoriensystem letztlich interpretativ absorbiert, sich selbst. Der realistische Standpunkt ist für die Explikation des Interpretationsgedankens - und nicht zuletzt für die Behauptung einer antirealistischen Sichtweise - logisch vorausgesetzt: „Pointiert formuliert: entweder wissen wir, was wir interpretieren, oder wir wissen gar nicht, dass wir interpretieren." (145) Lebensweltliche wie theoretische Erkenntnis ist letztlich realistisch verankert; nur vom Realismus her lässt sich dessen Negation oder die Abweichung von ihm artikulieren und verstehen. Die antirealistische Option, die unterstellt, „dass wir die Welt ,anders erkennen“, als sie tatsächlich ist“"(57), beruht auf einer ,petitio principii““ und positioniert sich in Wahrheit je schon im Horizont und auf der Basis des Realismus. Der Aufweis performativer Selbstwidersprüche, die sich mit einer konsequent interpretationistischen Position - beziehungsweise dem darin implizierten Subjektivismus, Konstruktivismus, Perspektivismus oder Skeptizismus - verbinden, bildet ein durchgehendes Motiv des Buchs von Krämer. Neben dem „Dilemma des Beweises“ des interpretationistischen Standpunktes (14 ff.) verweist Krämer auf ein analoges 
Dilemma der Argumentation und der Darstellung, sofern der Interpretationismus selbst seine Thesen ,auf allen Ebenen intentione recta“ (176) entfaltet.

Man kann sich fragen, wieweit die unter zahlreichen Abschattungen geführte Kritik am Antirealismus nicht nur die Interpretationsphilosophie, sondern die Hermeneutik als solche trifft. Zwei Punkte seien genannt. Zum einen ist zu fragen, wieweit die konstruktivistisch-perspektivistische Tendenz, die den Interpretationismus in prägnanten Formulierungen auszeichnet, eine eigene Stoßrichtung der Hermeneutik zu Ende führt oder sich zu dieser auch gegenläufig verhält. Abweichend von der durch Krämer vertretenen Lesart scheint es wichtig, den Gegenakzent nicht zu übersehen, den der hermeneutische Ansatz - zumal bei Gadamer - hier setzt. Die Gegenwendung zur Methodik der Naturwissenschaften führt nicht zu einem idealistisch-subjektzentrierten Erkennen. Ganz im Gegenteil beharrt Wahrheit und Methode nicht nur darauf, dass der „Fokus der Subjektivität ein Zerrspiegel“ (281) ist und nur die Zugehörigkeit zur Geschichte das Erkennen begründet. Die Vorbildfunktion von Kunst und Sprache steht auch dafür, dass Verstehen letztlich auf ein Ansichseiendes, von sich aus sich Mitteilendes geöffnet ist und Hermeneutik sich an der Vorgängigkeit des Objektiven - wenn auch nicht im Sinne wissenschaftlicher Tatsachenfeststellung - orientiert. Das durch Heidegger geprägte Verständnis der Wahrheit als Sichentbergen, das für die hermeneutische Wendung der Phänomenologie und die daran anschließende Philosophische Hermeneutik bestimmend ist, stellt gegenüber einer nietzscheanischen Theorie der Interpretationskonstrukte einen polaren Gegensatz dar. Die Ausrichtung der kritischen Auseinandersetzung um die Hermeneutik entlang der Trennlinie von Realismus und Antirealismus scheint nur zum Teil mit dem Anspruch und der genuinen Orientierung der Hermeneutik befasst.

Doch auch wenn man nicht auf das emphatische Wahrheitsverständnis von Wahrheit und Methode zurückgreift, bleibt fraglich, ob die gegenüber der Differenz von Natur- und Kulturwissenschaften indifferente epistemologische Problemstellung der Aufgabe einer HermeneutikKritik gerecht wird. Dabei geht es nicht darum, im Sinne traditioneller Verstehens- oder Einfühlungstheorien andere, weniger formale Modi des Erkennens und Erklärens geltend zu machen. Zur Diskussion steht die Differenz der Objektbereiche, die durch die konstitutive Reflexivität des Gegenstandes bedingt ist. Hermeneutik hat, auch wo sie nicht auf Texte und Zeichen beschränkt ist, mit Sinnhaftem zu tun, sofern der Bereich der Kultur - die Welt des objektiven Geistes, der Artefakte, der sozialen Lebensformen, der Geschichte - nicht unabhängig von den Beschreibungen und Deutungen zu definieren ist, die in sie eingegangen sind und den Sinn ausmachen, den diese Gegenstände für die in sie involvierten und von ihnen betroffenen Subjekte haben. Eine institutionelle Praxis kann nicht durch externe Verhaltensbeobachtung unabhängig vom Verständnis, das Subjekte mit ihr verbinden, beschrieben werden. Kulturwissenschaft ist eine Reflexion zweiter Stufe, die sich auf die dem Gegenstand immanente Selbstbeschreibung abstützt, doch sie nicht einfach wie eine gegenständliche Eigenschaft registriert, sondern mit ihr interagiert und damit gleichsam selbst in den Gegenstand eingeht. Zwischen Kunst und Kunstkritik, geschichtlichen Prozessen und historischer Reflexion, ökonomischen Mechanismen und Wirtschaftstheorien besteht ein kommunikativer Bezug, auf den sich Wissenschaft abstützt, ohne in ihm aufzugehen. Dies unterscheidet die , hermeneutische' Wissenschaft, die sich auf die Sinndimension ihres Gegenstandes einlässt, grundsätzlich von einer objektivistischen Beschreibung aus der Außenperspektive. Auf diesen Unterschied verweist die Betonung der ,Zugehörigkeit' zum Gegenstand, die gleichzeitig das Einrücken in eine Geschichte, das Teilnehmen an einem Prozess der Sinnbildung meint. Die Fundamentalität der Interpretation, welche hermeneutische und interpretationsphilosophische Ansätze teilen, meint sowohl das Ausgehen von den im Gegenstand sedimentierten Deutungen und Beschreibungen als auch das Eingehen der Interpretation in den Gegenstand und in die Prozessualität der menschlichen Welt. Dies ist der 
Horizont, innerhalb dessen die von Krämer facettenreich entfaltete Frage nach dem Realismus unseres Weltbezugs ihren Ort hat und in ihrer Intransigenz gestellt werden muss. 